\title{
Sarcopenia and Osteoporosis
}

\author{
Hyung-Min Ji, MD, Jun Han, MD, Ye-Yeon Won, MD, PhD \\ Department of Orthopedic Surgery, Ajou University Hospital, Ajou University School of Medicine, Suwon, Korea
}

Public health strategies designed to accomodate the ever-increasing human lifespan are urgently required. A good clinical understanding of frailty, as well as knowledge regarding how to prevent it, will therefore be required in order to overcome this challenge. Sarcopenia is an important component of the frailty syndrome, and its association with osteoporosis can lead to fractures and incident disability. Therefore, this review examined the literatuire pertaining to the association of sarcopenia with osteoporosis in order to assess preventive strategies.

Key Words: Aged, Frail elderly, Osteoporosis, Sarcopenia

\section{INTRODUCTION}

Rapid population aging poses serious social challenges worldwide. For example, unprecedented aging is progressing rapidly in Asian nations including Korea and Japan. Although the definition of an aged individual varies depending on the cultural and social environments of each country, an elderly person is defined as a person $\geq 65$ years or older, which aligns with the definition adopted by gerontologists ${ }^{1)}$. By this standard, Japan became a super-aged society in 2007 , as $22 \%$ of its population was $>65$ years old. Furthermore, Korea has been projected to become a super-aged society by $2026^{2}$. As we are witnessing today, population aging is an inevitable phenomenon occurring throughout the world

Submitted: May 25, 2015 1st revision: June 8, 2015

Final acceptance: June 8, 2015

Address reprint request to

Ye-Yeon Won, MD, PhD

Department of Orthopedic Surgery, Ajou University Hospital, Ajou University School of Medicine, 206 World cup-ro, Yeongtong-gu, Suwon 443-749, Korea

TEL: +82-31-219-5220 FAX: +82-31-219-5229

E-mail: thrtkr@ajou.ac.kr

This is an Open Access article distributed under the terms of the Creative Commons Attribution Non-Commercial License (http://creativecommons. org/licenses/by-nc/4.0) which permits unrestricted non-commercial use, distribution, and reproduction in any medium, provided the original work is properly cited. including Western countries and Japan, and is attributable to a complex mixture of economic, medical, educational and others factors. Consequently, aging poses social problems such as labor shortages and public health issues, such as osteoporotic fractures. The dramatic increase in the average life span in Korea has raised the public's interest regarding ways of not only living longer, but also reaching old age in good health. Therefore, healthy aging has also become a major public concern and active area of study in geriatric medicine, with an emphasis on the exploration of an array of strategies designed to improve quality of life of life in the elderly population ${ }^{3)}$. Instead of a traditional approach, which accepts a variety of pathological symptoms as natural consequences occurring in elderly populations, we introduce evedience to support a different theory; pathological symptoms as a series of consequences attrubutable to the frailty syndrome.

\section{MAIN SUBJECTS}

Frailty syndrome is a physiological state discriminated from normal aging or dysfunction. This syndrome occurs when the functioning of several organs diminishes and biological reserve declines below healthy levels. Unlike normal aging, however, frailty is characterized by increased vulnerability to several disorders after exposure to the same stressful situation ${ }^{4,5)}$. 
Even though frailty is a common syndrome in older adults, it is not an inevitable part of the aging process, as only a segment of the elderly population experience this syndrome. Although several frailty-defining criteria have been proposed, frailty has been defined as meeting three of the five criteria presented in Table 1. Additionally, a pre-frail stage has been defined as meeting one or two of the criteria ${ }^{5}$. Not only is frailty considered a clinical syndrome that can be discriminated distinctively from normal aging and progressive physiological changes, but it is also one of the stages in the progression from normal aging to the pre-frail stage, subsequent frailty syndrome, and ultimately, geriatric disease itself ${ }^{4}$. The clinical manifestations of frailty are weight loss, sarcopenia, low physical activity, a diminished sense of balance and gait speed, diminished cognitive functioning, and nutritional deficiency. Therefore, frailty carries an increased risk for poor functioning in activities of daily living including locomotive abilities, the incidence of cardiovascular

Table 1. Definition of Frailty Syndrome

The presence of 3 or more of the following symptoms

1) Unintentional weight loss (4-5 kg in 1 year)

2) Self reported exhaustion

3) Weakness (grip strength $<20 \%$ in the dominant hand)

4) Slow walking speed ( $<20 \%$ for time to walk $4.5 \mathrm{~m}$ )

5) Low physical activity ( $<20 \%$ for calorie expenditure) diseases, cancers, falls, and mortality ${ }^{6}$.

The results from a large number of studies verified that frailty was profoundly related to osteopenia or osteoporosis, sarcopenia, and falls ${ }^{6}$. As the clinical definition of frailty includes the diagnostic criteria for sarcopenia, one must be familiar with the definition of sarcopenia itself, which is the consistent and systematic loss of skeletal muscle mass and strength prevalent in older adults ${ }^{7}$. Rosenberg ${ }^{8)}$ first proposed the term 'sarcopenia' (from the Greek 'sarx' for flesh and 'penia' for loss) to describe the observed age-associated decrease in muscle mass. When dual-energy X-ray absorptiometry (DEXA), computed tomography (CT), magnetic resonance imaging (MRI), and other new radiological instruments began to appear, it became possible to measure relative masses according to human body compositions, such as fat, water, minerals, and skeletal muscle. There is little doubt that skeletal muscle mass decreases with aging, and it has been hypothesized that falls and fractures in the elderly are attributable to this loss of skeletal muscle mass. To date, a number of studies have been conducted in order to examine different aspects of the decline in skeletal muscle mass and strength using various methods,all of which verified that sarcopenia resulted in diminished vitality and quality of life and increased the risk of mortality ${ }^{6}$. In 2010, European researchers developed diagnostic guidelines for sarcopenia ${ }^{7}$, which is initially determined by a decreased amount of muscle mass, followed by reduced levels of muscle strength and physical activity

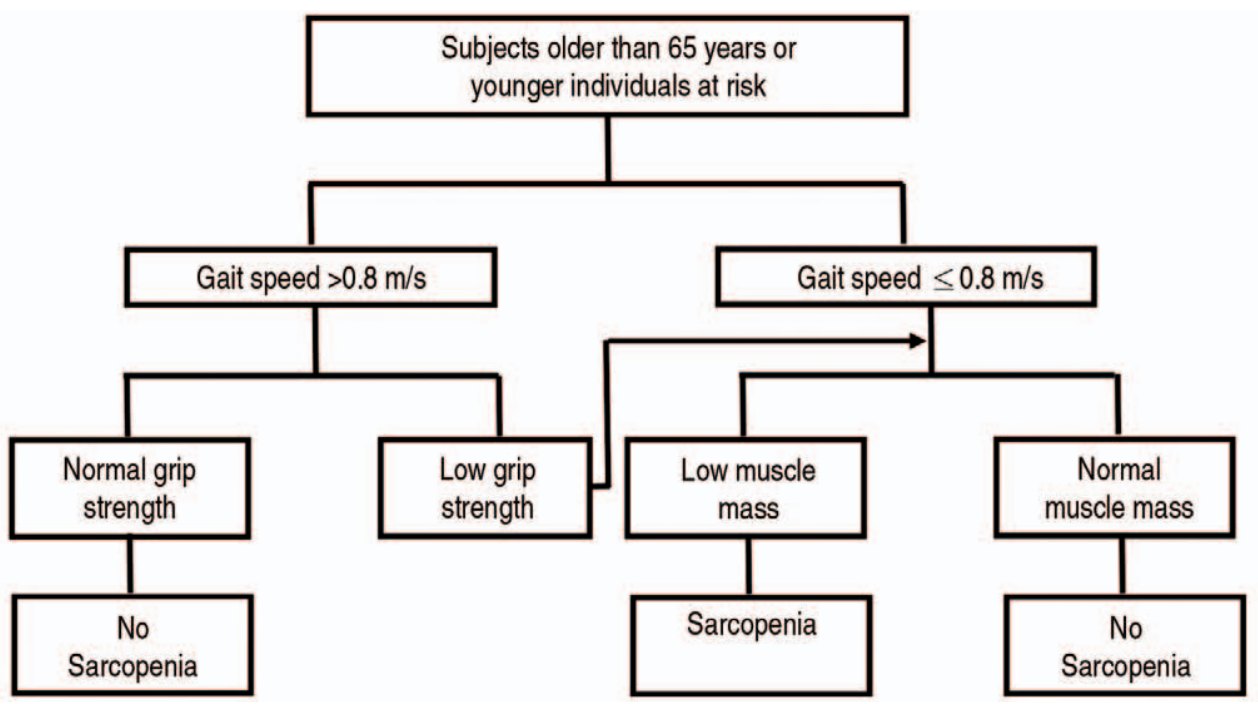

Fig. 1. European Working Group on Sarcopenia in Older People-suggested algorithm for sarcopenia case finding in older individuals. 
(Fig. 1). To measure the size of muscles, relative skeletal muscle mass also has to be measured using DEXA, CT, and MRI, among others. In relevant studies, the relative muscle mass was calculated as the total body skeletal mass divided by squared height ${ }^{9)}$ or weight ${ }^{10)}$. Although the diagnostic criteria varied slightly across the different studies, class II sarcopenia was typically defined as a skeletal mass index $<2$ standard deviations below mean values for young adults, and was considered to be clinically significant. The standard values of Korean men and women in domestic studies were used as a reference base for the reviewed studies ${ }^{11}$.

According to the results of a cross-sectional study, older adults had a $25-35 \%$ smaller lower extremity muscle mass than younger adults, and age-related loss of muscle mass occured at a rate of $1 \%$ annually, dropping continuously up to $40 \%$ smaller between those in their $20 \mathrm{~s}$ and those in their $60 \mathrm{~s}^{3}$. Lower extremity muscle strength decreased at a rate of $3 \%$ annually, and the muscle power of older adults was $20-40 \%$ lower than that of younger adults ${ }^{12}$. When comparing muscle strength differences between men and women, the rate of muscle loss was found to be somewhat similar, but the absolute reduction of muscle mass was observed to be greater in men $^{3)}$. In order to accurately measure muscle power, it is ideal to measure knee extensor and flexor strength. Because this method is not applicable to all patients, grip strength has been a commonly applied measured due to the suggestion that it can be used to relatively and precisely represent lower extremity muscle power ${ }^{13)}$.

Gait speed has been measured to assess physical activity levels, and a low gait speed (defined as a walking speed $<0.8 \mathrm{~m} / \mathrm{s}$ ) is part of the diagnostic criteria for sarcopenia according to the European Working Group on Sarcopenia in Older People ${ }^{7}$. More recently, some Asian investigators have proposed that a walking speed $<1-1.2 \mathrm{~m} / \mathrm{s}$ would be more appropriate than the European standards ${ }^{1)}$. Furthermore, other useful methods have been suggested including the short physical performance battery and the timed-up-and-go test. According to Asian clinicians, however, measuring gait speed using a walking distance of $6 \mathrm{~m}$ is considered as a repeatable and practical measure.

According to the findings derived from a metaanalysis, sarcopenia was found to be the root cause of falls in a a vriety of studies, increasing the risk of falls by up to 1.5-3.0 fold ${ }^{14)}$. Moreover, the results of a previous study demonstrated that the weakened strength of the upper limb muscles increased the risk of falls by up to 1.4-1.5 fold ${ }^{14)}$. Therefore, the loss of muscle strength is an important indicator for predicting the incidence of falls. Additionally, the results of biomechanical studies and electromyography have revealed that older individuals with sarcopenia had slower contraction and response rates in their lower limb muscles ${ }^{15}$, and that muscle strength had declined ${ }^{16}$. Although body balance is more easily restored in younger people, it my not be maintained properly in older individuals and can influence thier propensity to fall.

Sarcopenia is associated with diminished physical activity levels, incident disability, and is a major component of frailty. Collectivelly, these findings may explain the pathologic mechanisms underlying sarcopenia as a leading cause of higher rates of frailty. The results of previous studies have indicated that the cross section muscle of the thigh, the degree of fat infiltration, and muscle strength, among others, were vital factors for predicting incident disability, and that fat infiltration and muscle power were more crucial criteria than muscle mass ${ }^{17}$. Regardless of bone mineral density (BMD), the degree of fatty infiltration in muscles has been found to increase the risk of hip fractures ${ }^{18}$. Additional studies exploring the role of sarcopenia in relation to the aforementioned finding therefore seem to be warranted. As recent findings have revealed that muscle fat infiltration can be caused by obesity, sarcopenic obesity has been drawing increased attention ${ }^{19}$.

The function and formation of bones and muscles are closely related ${ }^{20)}$, and skeletal shape is determined by loads changing as muscle strength and size increase ${ }^{21}$. As a result of muscle loss occurring after the age of 50 years, the workload imposed on the skeleton gradually decreases, and atrophy of bone-muscle unit can be observed. The age-related loss of bone and muscle mass occurs almost simultaneously, and the loss of muscle mass is predominantly detected in type II fibers. Consequently, the entire bone-muscle unit is reduced to $50 \%$ in elderly adults as compared with young adults. Additionally, the quality and fine structures of the bones are deteriorated. These two biological outcomes involve similar hormonal changes, and abnormal hormonal effects on the growth hormone/insulin-like growth factor-1/phosphoinositide 3-kinase/Akt pathway has been suggested to have the potential to induce the 
osteoporosis associated with sarcopenia ${ }^{22)}$.

It has been speculated that vitamin $\mathrm{D}$ may be one of the mediators of muscle and bone weakening, and this assertion was validated by the results of a study that found patients with decreased vitamin D levels experienced muscle and bone weakening ${ }^{23}$. Due to the results generated from a number of recent studies which indicated that vitamin D was primarily involved in physiological changes in the bones and muscles, as well as those showing that vitamin D insufficiency was a main contributor to sarcopenia and osteoporosis, the roles of vitamin D have drawn increased attention. The results from a previous study suggested that sarcopenia and osteoporosis acted as a kind of 'hazardous duet', eventually leading to frailty ${ }^{24,25}$. However, it remained uncertain as to whether the interaction of osteoporosis and inadequate skeletal muscle mass affected frailty, or whether the separate conditions made individual contributions. The aforementioned study reported that frailty was six times more likely to occur in patients with both conditions, strongly suggesting that the interaction of the two conditions contributes to frailty. Alternatively, treating osteoporosis or increasing muscle mass can be solutions to the problem. The results of one study indicated that an increase in BMD and sarcopenia treatment could be achieved concurrently through osteoporosis management ${ }^{26}$. Moreover, the results of a study involving a muscle building workout regimen indicated a simultaneous increase in BMD, supporting this alternate hypothesis ${ }^{27)}$. However, contradictory results regarding whether the combination of osteoporosis and sarcopenia played a significant role in the problem in clinical trials ${ }^{19)}$ suggest that further studies are warranted.

\section{CONCLUSION}

The development of a broad array of medications, as well as the clinical trials associated with these medications, are currently in progress. These efforts are expected to provide new options for the treatment of osteoporosis and sarcopenia. The immediate goals of such research are to develop the effective strategies required to overcome geriatric frailty, which can only be found through medical research, treatment, and a multidisciplinary approach.

\section{REFERENCES}

1. Chen LK, Liu LK, Woo J, et al. Sarcopenia in Asia: consensus report of the Asian Working Group for Sarcopenia. J Am Med Dir Assoc. 2014;15:95-101.

2. Korea National Statistical Office. Prediction of future population change, 2006. Daejeon: Statistics Korea; 2006.

3. Lang T, Streeper T, Cawthon P, Baldwin K, Taaffe DR, Harris TB. Sarcopenia: etiology, clinical consequences, intervention, and assessment. Osteoporos Int. 2010;21:543 -59 .

4. Park BJ, Lee YJ. Integrative approach to elderly frailty. Korean J Fam Med. 2010;31:747-54.

5. Fried LP, Ferrucci L, Darer J, Williamson JD, Anderson G. Untangling the concepts of disability, frailty, and comorbidity: implications for improved targeting and care. J Gerontol A Biol Sci Med Sci. 2004;59:255-63.

6.Cruz-Jentoft AJ. Perspective: Protein and exercise for frailty and sarcopenia: still learning. J Am Med Dir Assoc. 2013;14:69-71.

7. Cruz-Jentoft AJ, Baeyens JP, Bauer JM, et al; European Working Group on Sarcopenia in Older People. Sarcopenia: European consensus on definition and diagnosis: Report of the European Working Group on Sarcopenia in Older People. Age Ageing. 2010;39:412-23.

8. Rosenberg IH. Sarcopenia: origins and clinical relevance. J Nutr. 1997; 127(5 Suppl):990S-1S.

9. Baumgartner RN, Koehler KM, Gallagher D, et al. Epidemiology of sarcopenia among the elderly in New Mexico. Am J Epidemiol. 1998;147:755-63.

10. Janssen I, Heymsfield SB, Ross R. Low relative skeletal muscle mass (sarcopenia) in older persons is associated with functional impairment and physical disability. J Am Geriatr Soc. 2002;50:889-96.

11. Kim YS, Lee Y, Chung YS, et al. Prevalence of sarcopenia and sarcopenic obesity in the Korean population based on the Fourth Korean National Health and Nutritional Examination Surveys. J Gerontol A Biol Sci Med Sci. 2012;67:1107-13.

12. Hughes VA, Frontera WR, Wood M, et al. Longitudinal muscle strength changes in older adults: influence of muscle mass, physical activity, and health. J Gerontol A Biol Sci Med Sci. 2001;56:B209-17.

13. Aadahl M, Beyer N, Linneberg A, Thuesen BH, Jørgensen T. Grip strength and lower limb extension power in 19-72year-old Danish men and women: the Health2006 study. BMJ Open. 2011;1:e000192.

14. Moreland JD, Richardson JA, Goldsmith CH, Clase CM. Muscle weakness and falls in older adults: a systematic review and meta-analysis. J Am Geriatr Soc. 2004;52:1121-9.

15. Thelen DG, Muriuki M, James J, Schultz AB, AshtonMiller JA, Alexander NB. Muscle activities used by young and old adults when stepping to regain balance during a forward fall. J Electromyogr Kinesiol. 2000;10:93-101.

16. Wojcik LA, Thelen DG, Schultz AB, Ashton-Miller JA, Alexander NB. Age and gender differences in peak lower extremity joint torques and ranges of motion used during single-step balance recovery from a forward fall. J Biomech. 2001;34:67-73. 


\section{Hip \& Pelvis}

Hip Pelvis 27(2): 72-76, 2015

17. Visser M, Goodpaster BH, Kritchevsky SB, et al. Muscle mass, muscle strength, and muscle fat infiltration as predictors of incident mobility limitations in wellfunctioning older persons. J Gerontol A Biol Sci Med Sci. 2005;60:324-33.

18. Lang T, Cauley JA, Tylavsky F, Bauer D, Cummings S, Harris TB; Health ABC Study. Computed tomographic measurements of thigh muscle cross-sectional area and attenuation coefficient predict hip fracture: the health, aging, and body composition study. J Bone Miner Res. 2010;25:513-9.

19. Tarantino U, Piccirilli E, Fantini M, Baldi J, Gasbarra E, Bei R. Sarcopenia and fragility fractures: molecular and clinical evidence of the bone-muscle interaction. J Bone Joint Surg Am. 2015;97:429-37.

20.DiGirolamo DJ, Kiel DP, Esser KA. Bone and skeletal muscle: neighbors with close ties. J Bone Miner Res. 2013;28:1509-18.

21. Tarantino U, Baldi J, Celi M, et al. Osteoporosis and sarcopenia: the connections. Aging Clin Exp Res. 2013;25 Suppl 1:S93-5.
22. Terracciano C, Celi M, Lecce D, et al. Differential features of muscle fiber atrophy in osteoporosis and osteoarthritis. Osteoporos Int. 2013;24:1095-100.

23. Gunton JE, Girgis CM, Baldock PA, Lips P. Bone muscle interactions and vitamin D. Bone. Published online March, 2015; doi: 10.1016/j.bone.2015.02.029.

24. Frisoli A Jr, Chaves PH, Ingham SJ, Fried LP. Severe osteopenia and osteoporosis, sarcopenia, and frailty status in community-dwelling older women: results from the Women's Health and Aging Study (WHAS) II. Bone. 2011; 48:952-7.

25. Crepaldi G, Maggi S. Sarcopenia and osteoporosis: A hazardous duet. J Endocrinol Invest. 2005;28(10 Suppl):66-8.

26. Park JH, Park KH, Cho S, et al. Concomitant increase in muscle strength and bone mineral density with decreasing IL-6 levels after combination therapy with alendronate and calcitriol in postmenopausal women. Menopause. 2013;20:747-53.

27. Suominen H. Muscle training for bone strength. Aging Clin Exp Res. 2006;18:85-93. 